\title{
NMSPEC: A Fortran code for the sparticle and Higgs masses in the NMSSM with GUT scale boundary conditions
}

\author{
Ulrich Ellwanger \\ Laboratoire de Physique Théorique ${ }^{1}$ \\ Université de Paris XI, F-91405 Orsay Cedex, France \\ Cyril Hugonie \\ Laboratoire Physique Théorique et Astroparticules ${ }^{2}$ \\ Université de Montpellier II, F-34095 Montpellier, France
}

\begin{abstract}
NMSPEC is a Fortran code that computes the sparticle and Higgs masses, as well as Higgs decay widths and couplings in the NMSSM, with soft SUSY breaking terms specified at $M_{\mathrm{GUT}}$. Exceptions are the soft singlet mass $m_{s}^{2}$ and the singlet self coupling $\kappa$, that are both determined in terms of the other parameters through the minimization equations of the Higgs potential. We present a first analysis of the NMSSM parameter space with universal SUSY breaking terms at $M_{\mathrm{GUT}}$ - except for $m_{s}$ and $A_{\kappa}$ - that passes present experimental constraints on sparticle and Higgs masses. We discuss in some detail a region in parameter space where a SM-like Higgs boson decays dominantly into two CP odd singlet-like Higgs states.
\end{abstract}

PAC numbers: 12.60.Jv, 14.80.Cp, 14.80.Ly

December 2006

LPT Orsay 06-79

LPTA Montpellier 06-61

\footnotetext{
${ }^{1}$ Unité mixte de Recherche - CNRS - UMR 8627

${ }^{2}$ Unité mixte de Recherche - CNRS - UMR 5207
} 


\section{Introduction}

The Next to Minimal Supersymmetric Standard Model (NMSSM) [1,2] provides a very elegant solution to the $\mu$ problem of the MSSM via the introduction of a singlet superfield $S$. For the simplest possible scale invariant form of the superpotential, the scalar component of $S$ acquires naturally a vacuum expectation value of the order of the SUSY breaking scale, giving rise to a value of $\mu$ of order the electroweak scale. Hence the NMSSM is the simplest supersymmetric extension of the standard model in which the fundamental Lagrangian contains just SUSY breaking terms but no other parameters of the order of the electroweak scale.

As in the MSSM, the phenomenology of the NMSSM depends on a certain number of parameters (mostly soft SUSY breaking parameters) that cannot be predicted from an underlying theory at present. It is then useful to have computer codes that compute physically relevant quantities as Higgs and sparticle masses, couplings, decay widths etc. as functions of the initial parameters in the Lagrangian. Such codes allow to investigate which regions in parameter space are in conflict with present constraints on physics beyond the standard model and, most importantly, which regions in parameter space can be tested in future experiments and/or astrophysical measurements. A review over corresponding publicly available computer codes can be found in [3].

Most of these codes are limited to the MSSM; the only presently available code for the NMSSM is NMHDECAY $[4,5]$.

Many mechanisms for spontaneous SUSY breaking (such as supergravity with universal kinetic terms for all matter fields) predict a simple pattern for the soft susY breaking parameters at a large scale as the GUT scale. Then, the soft sUSY breaking parameters are often universal, i.e. completely specified by universal gaugino masses, scalar masses squared and trilinear couplings. In the case of the MSSM, the corresponding scenario is denoted as the CMSSM (or mSUGRA since, in principle, other scenarios as GMSB or AMSB could constrain the soft SUSY breaking parameters). The advantage of such a scenario is, apart from its motivation through a simple underlying theory, obviously an enormous reduction of the number of unknown parameters. It would be desirable, if one could implement such simple boundary conditions also in the NMSSM, which we will denote as the CNMSSM.

Numerical studies of the sparticle and Higgs spectrum in the CNMSSM have been performed in [2]. Since then, additional radiative corrections to the Higgs spectrum have been computed [6], and experimental constraints on the sparticle and notably the Higgs spectrum have become considerably stronger [7]. Also, the corresponding numerical codes have not 
been made publicly available.

NMSPEC is a code that allows to chose parameters for the NMSSM at the GUT scale. Exceptions are the singlet self coupling $\kappa$ and the soft SUSY breaking singlet mass $m_{s}^{2}$; as described below, these two parameters are determined in terms of others through the minimization equations of the Higgs potential. Possible scenarios such as GMSB or AMSB are not (yet) implemented in NMSPEC. Also, like NMHDECAY, NMSPEC is at present limited to the simplest version of the NMSSM; neither terms linear in the singlet [8], additional U(1) gauge symmetries [9] or combinations thereof [10] are considered. (See [11] for a review of such more general versions of the NMSSM.) Subsequently we limit ourselves to a scale invariant superpotential of the form

$$
W=\lambda S H_{u} H_{d}+\frac{\kappa}{3} S^{3}+\ldots,
$$

and the associated soft SUSY breaking trilinear couplings $A_{\lambda}, A_{\kappa}$ and Higgs masses.

In principle numerical codes, that are designed to compute the particle and sparticle spectrum depending on the soft SUSY breaking terms defined at the GUT scale, could proceed as follows: starting with numerical values for all unknown parameters at $M_{\mathrm{GUT}}$, the $\mathrm{RG}$ equations can be integrated numerically down to the weak scale. Then, the effective Higgs potential can be minimized numerically, and from the Higgs vevs at the minimum one obtains $\tan \beta$ and $M_{Z}$ (where $M_{Z}$ is used to determine a previously unspecified overall mass scale). This approach has been used for the NMSSM in [2].

It has several disadvantages, however: First, the numerical minimization of the Higgs potential is quite computer time consuming. Second, since the overall mass scale and $\tan \beta$ are determined only at the end, one finds that $m_{\text {top }}$, Higgs and sparticle masses often violate experimental bounds - it is not possible to input "large" soft terms (and the correct value of $h_{t}$ ) from the start.

An advantage of this approach would be that it makes required (fine tuned) relations among the initial parameters immediately obvious.

However, in view of its disadvantages, practically all present numerical codes (within the MSSM) proceed differently: both $M_{Z}$ and $\tan \beta$ are used as inputs, and the two minimization equations of the Higgs potential w.r.t. the two real Higgs vevs $H_{u}$ and $H_{d}$ are used to compute $\mu$ and the soft $B$ parameter in terms of the others. (This leaves open the sign of $\mu$; subsequently the two possibilities have to be treated separately.) $\mu$ and $B$ have only a small effect on the RG evolution of the other parameters, only via threshold effects from particles whose masses depend on $\mu$ and/or $B$. One can take care of this via a (typically rapidly converging) iterative procedure. (Now, however, possible fine tunings originating notably 
from the requirement to chose $B$ very precisely, are hidden.)

At first sight, an application of this procedure to the NMSSM is not obvious: both $\mu$ and $B$ are no longer independent parameters (although effective $S$ dependent parameters $\mu_{\mathrm{eff}}=\lambda S, B_{\mathrm{eff}}=A_{\lambda}+\kappa S$ can still be defined), and one has to cope with three coupled minimization equations w.r.t. $H_{u}, H_{d}$ and $S$.

A possible way out is the following: First, the tree level minimization equations w.r.t. $H_{u}$ and $H_{d}$ can be solved for $\mu_{\text {eff }}$ and $B_{\text {eff }}$, as in the MSSM, in terms of the other parameters (incl. $M_{Z}$ and $\tan \beta$ ). From $\mu_{\text {eff }}$ and $B_{\text {eff }}$ one can deduce (for $\lambda$ and $A_{\lambda}$ given) both $S$ and $\kappa$. Finally, from the minimization equation w.r.t. $S$, one can easily obtain the soft singlet mass $m_{s}^{2}$ in terms of all other parameters.

This allows to chose as input parameters in the Higgs sector of the NMSSM the soft Higgs doublet masses squared, $A_{\lambda}, A_{\kappa}, M_{Z}, \tan \beta$ and $\lambda$. From these the three parameters $\mu_{\text {eff }}$ (or $S), \kappa$ and $m_{s}^{2}$ can be derived. The radiative corrections to the Higgs potential (that depend on all sparticle and Higgs masses) show a weak dependence on these parameters. They can be included in the minimization equations that become non-linear in the parameters to solve for, but one can solve them iteratively by a loop that converges rapidly.

The derived parameters $\kappa$ and $m_{s}^{2}$ affect the RG evolution equations of some of the other parameters not only through threshold effects around $M_{\text {susy }}$, but through the $\beta$ functions (already at one loop). However, the numerical impact is relatively small such that an iterative procedure converges quite rapidly again. (These iterative procedures are described in section 2.)

Clearly, once $m_{s}^{2}$ is an output rather than an input, it becomes difficult to find parameters such that $m_{s}^{2}$ at the GUT scale assumes the same value as, e.g., the Higgs doublet (or other scalar) soft masses squared. On the other hand it is easy to imagine that a mecanism for the generation of soft SUSY breaking terms treats the singlet differently from the other non-singlet matter multiplets.

It happens frequently that the derived value of $m_{s}^{2}$ is negative even at the GUT scale. This does not imply that the singlet vev takes values of the order of the GUT scale, or that the scalar potential is unbounded from below: due to the term $\kappa^{2} S^{4}$ in the scalar potential, the singlet vev will always be of the order of $m_{s} / \kappa$ (hence of the order of $M_{\text {susy }}$ ), and it is still necessary to integrate the RG equations also for the couplings and masses involving the singlet down to the SUSY scale and to compute further radiative corrections there.

Once $\kappa$ is an output rather than an input, it is difficult to study the "Peccei-Quinn" limit $\kappa \rightarrow 0$ (with $\lambda$ finite) in the NMSSM [12]. However, this limit is quite unphysical at least in the simplest version of the NMSSM considered here: Once $\kappa$ tends to zero, the singlet 
vev $S$ tends to infinity, and hence $\mu_{\text {eff }}=\lambda S$ becomes unacceptably large: large $\mu_{\text {eff }}$ implies a stable Higgs doublet potential at the origin (hence no electroweak symmetry breaking), unless at least one of the Higgs doublet SUSY breaking masses squared is of the same order and negative. Hence $\kappa \rightarrow 0$ would require $M_{\text {susy }} \rightarrow \infty$.

The limit where $\kappa$ and $\lambda$ tend to zero simultaneously and the NMSSM turns into the MSSM plus a decoupled singlet sector poses no problem, however: it is easy to see that, for given and fixed $\mu_{\mathrm{eff}}, B_{\mathrm{eff}}$ and $A_{\lambda}, \lambda \rightarrow 0$ implies automatically $\kappa \sim \lambda \rightarrow 0$. This limit is also stable under the RG flow from the GUT scale down to the electroweak scale. Although the vev $S$ tends towards infinity in this limit, the masses of all components of the singlet superfield remain of the order of $M_{\text {susy }}$ (i.e. of the order of $A_{\kappa}, A_{\lambda}$ and $\mu_{\text {eff }}$ ). Hence, in order to study the MSSM limit of the NMSSM within NMSPEC, it is enough to chose a tiny value for $\lambda$ on input. ( $\lambda=0$ is not allowed, since expressions proportional to $\kappa / \lambda$ would become ill defined. Also, $A_{\kappa}$ still has to be chosen within a $\mu_{\mathrm{eff}}$ and $A_{\lambda}$ dependent window in order to avoid negative masses squared for the CP even and/or the CP odd singlet scalars.)

Unless modified by the user, NMSPEC allows to chose the following input parameters: $\tan \beta$ and the sign of $\mu_{\text {eff }}$ at the weak scale and $\lambda$ at the SUSY scale; at the GUT scale the free parameters are universal gaugino masses $M_{1 / 2}$, universal scalar masses $m_{0}^{2}$ and universal trilinear couplings $A_{0}$. Exceptions are the following:

a) the soft singlet mass at $M_{\mathrm{GUT}}$ is an output, as described above;

b) the trilinear coupling $A_{\kappa}$ can be chosen differently from the other trilinear couplings. The reason for this is twofold: first, if an underlying mecanism for the generation of the soft SUSY breaking terms treats the singlet differently from the other matter fields (as it is already assumed for $m_{s}^{2}$ ), this will also affect the coupling $A_{\kappa}$ which involves singlets only. (We left $A_{\lambda}$ unified with the other trilinear couplings at $M_{\mathrm{GUT}}$; this could easily be changed by the user, however.)

Also, interesting physics is associated with particular values of $A_{\kappa}$ : in certain regions of the parameter space of the NMSSM, the lightest neutral CP even doublet-like Higgs boson (the one ressembling to the SM Higgs boson) can decay into two lighter neutral CP odd (sometimes also CP even) Higgs bosons [13,14]. This process allows for a doubletlike CP even Higgs boson $h$ to escape LEP constraints even for $m_{h}$ below $114 \mathrm{GeV}$. (The corresponding LEP constraints have recently been updated in [7], see the discussion in section 3 below. The allowed window has become somewhat tighter, but still exists.) On the one hand, such scenarios can make the detection of a CP even Higgs boson at the LHC quite difficult $[13,14]$. It has also been argued that the fine tuning, that is required in the MSSM in order to lift the Higgs mass above the LEP bound, is relieved in the corresponding region 
of parameters in the NMSSM [14]. This region of parameters in the NMSSM requires, however, that $A_{\kappa}$ assumes values within a certain range such that singlet-like Higgs bosons are sufficiently light. The possibility to chose $A_{\kappa}$ differently from the other trilinear couplings in NMSPEC allows to study this range (now in terms of $A_{\kappa}$ defined at $M_{\mathrm{GUT}}$ ), and the physics associated with it.

In the next section 2 we describe in detail, how the code NMSPEC deals with the various input parameters in the NMSSM. In section 3 we discuss new features of some of the subroutines of NMHDECAY v3; most of the subroutines of NMHDECAY v3 are shared by NMSPEC. In section 4 we present first results obtained with NMSPEC. The allowed parameter space (passing all phenomenological tests) divides into three regions: a MSSM-like region with $\lambda$ relatively small, and two NMSSM specific regions with $\lambda$ large either for small $\tan \beta$ and $m_{h}$ above $114 \mathrm{GeV}$, or for medium to large $\tan \beta$ and $m_{h}$ below $114 \mathrm{GeV}$, but $A_{\kappa}$ within a certain window that renders the unconventional Higgs decay modes discussed above possible. The width of the corresponding window (and the fine tuning associated with it) is discussed in some detail.

\section{The code NMSPEC}

As discussed in the introduction, the code NMSPEC proceeds as follows: Input parameters are the SM gauge couplings, Fermion masses of the 3rd generation, $\tan \beta$, the sign of $\mu_{\text {eff }}$, and $\lambda$ as well as the soft SUSY breaking masses and trilinear couplings (with the exception of $m_{s}^{2}$ ) at the GUT scale.

The code NMSPEC proceeds as follows: First, a guess for $\kappa, m_{s}^{2}, \mu_{\text {eff }}$ and $M_{\text {susy }}$ is made, and a preliminary calculation of $M_{\mathrm{GUT}}$ and the gauge and Yukawa couplings at the GUT scale is performed through an integration of the (two loop) RG equations from the SUSY scale up to $M_{\mathrm{GUT}}$ using the subroutine RGES. ( $M_{\mathrm{GUT}}$ is defined as the scale where $\alpha_{1}$ and $\alpha_{2}$ unify.)

Then, an "external" loop starts, that involves both the GUT scale and $M_{\text {susy }}$ : the (two loop) RG equations for the soft terms, together with the gauge and Yukawa couplings, are integrated from $M_{\mathrm{GUT}}$ down to $M_{\text {susy }}$ in the subroutine RGESINV.

There an "internal" loop starts, that involves physics between $M_{\text {susy }}$ and the weak scale:

In the subroutine RUNPAR, first the mass $M_{A}^{2}$ of a potentially heavy Higgs doublet is estimated (which is required for the threshold effects below). The susy scale (denoted by Q2) is defined in terms of the squark masses of the first generations, and a scale QSTSB is defined in terms of the left and right stop masses squared. Then, the SM gauge and 
Yukawa couplings are calculated at the scale QSTSB (together with the Higgs wave function renormalization constants $Z_{i}$ ). Threshold effects at Higgs and sparticle masses are taken care of. Finally, the NMSSM specific Yukawa and trilinear couplings are computed at QSTSB.

In the next subroutine MSFERM, the ( $\left.\mu_{\text {eff-dependend }}\right) 3$ rd generation squark and slepton pole masses and mixing angles are computed at the scale QSTSB. They are needed below for the radiative corrections to the minimization equations of the Higgs potential.

In the following subroutine LOWMUK, first the RG equations for the soft Higgs doublet masses squared are integrated from $Q 2$ to QSTSB. Then, $\mu_{\text {eff }}, \kappa$ and $m_{s}^{2}$ are determined from the minimization equations of the Higgs potential including radiative corrections, that depend on previous estimates for $\mu_{\text {eff }}$ and $\kappa$. A variable CHECK verifies the relative change of $\mu_{\text {eff }}$ and $\kappa$ with respect to its previous values. Finally, an improved value for $M_{A}^{2}$ is computed (in case the internal loop stops here; otherwise its previous estimate is used again).

Unless CHECK $<10^{-6}$, the internal loop returns now to the subroutine RUNPAR. There, the improved values for $\mu_{\text {eff }}$ and $\kappa$ are used for improved threshold corrections. In the next call of LOWMUK, the radiative corrections to the minimization equations also include the improved values of $\mu_{\text {eff }}$ and $\kappa$.

If CHECK $<10^{-6}, \mu_{\text {eff }}$ and $\kappa$ have been determined to a sufficient accuracy. We have never observed a convergence problem related to the determination of $\mu_{\text {eff }}$ and $\kappa ; 5$ inner loops are usually more than sufficient.

Then, the "external" loop continues: next, the RG equations for the SM gauge and Yukawa couplings are integrated again from the weak scale up to $M_{\mathrm{GUT}}$ in the subroutine RGES, using improved threshold effects at sparticle and Higgs masses as well as the updated value for $\kappa$. The value for $M_{\mathrm{GUT}}$ is improved simultaneously.

Next, the RG equations for the soft terms are integrated from $M_{\text {susy }}$ up to $M_{\mathrm{GUT}}$ in the subroutine RGESUNI, using the new SM gauge and Yukawa couplings and the new values for $M_{\mathrm{GUT}}$ and $m_{s}^{2}$. A variable GUTEST verifies, whether all soft terms coincide with the required boundary conditions at $M_{\mathrm{GUT}}$.

Unless the relative deviation determined by GUTEST is less than $10^{-4}$ (for all soft SUSY breaking parameters), the external loop jumps back to a call of RGESINV. There, the new values for $M_{\mathrm{GUT}}$ and the SM gauge and Yukawa couplings are used in order to integrate again the RG equations for the soft terms (with the required boundary conditions at $M_{\mathrm{GUT}}$ ) down from the GUT scale.

If GUTEST is less than $10^{-4}$, the required values of the soft terms at $M_{\mathrm{GUT}}$ are considered as sufficiently precise, and the external loop is left. The subsequent subroutines of NMSPEC are shared with NMHDECAY v3: Higgs, gluino, chargino and neutralino masses are computed, 
and all Higgs branching ratios into SM particles and sparticles are determined. Then, present experimental constraints on Higgs and sparticles masses and couplings are verified. Warnings indicate at the end, whether such constraints are violated. A rough (1 loop) computation of the $b \rightarrow s \gamma$ branching ratio is performed (for information only, without generating an error message), and it is verified whether the choice of parameters corresponds to a global minimum of the NMSSM Higgs potential.

Finally, if desired (if the flag OMGFLAG is on), the dark matter relic density is computed using a NMSSM version of the micrOMEGAs_2.0 subroutines [15]. These subroutines are called internally in NMHDECAY v3 and NMSPEC; an interface file, as it was the case for NMHDECAY v2 [16], is not needed anymore. The NMSSM version of micrOMEGAs_2.0 is included in the downloadable package NMSSMTools_1.0, and compiled together with NMHDECAY and NMSPEC, as described in the appendix.

The different options (a single point in parameter space, scans over regions in parameter space using grids or random numbers) are also described in the appendix.

\section{New features in NMHDECAY v3 and NMSPEC}

The code NMHDECAY v3 performs the same tasks as the codes NMHDECAY v1 and v2, that are described in detail in $[4,5]$. Now, many subroutines are shared by NMSPEC, and the codes NMHDECAY v3 and NMSPEC can be downloaded simultaneously (see the appendix). Apart from the architecture, some properties of some subroutines have been improved with respect to NMHDECAY v2; these changes are described below.

\subsection{Experimental constraints}

The (negative) results of the four LEP collaborations ALEPH, DELPHI, L3 and OPAL on Higgs Boson searches have been updated and combined by the LEP Higgs Working group in [7]. As compared to the corresponding constraints already implemented in NMHDECAY v2, the updates in [7] include improved constraints on Higgs masses vs. couplings for the following processes:

- $Z \rightarrow Z H_{2} \rightarrow Z H_{1} H_{1}$ where $H_{1}$ stands for a $\mathrm{CP}$ even or a CP odd neutral Higgs boson. The considered decay channels of $H_{1}$ are $H_{1} \rightarrow 2 b$ and $H_{1} \rightarrow 2 \tau$, and combinations thereof.

- $Z \rightarrow H_{1} H_{2}$ where both $H_{1}$ and $H_{2}$ can denote a CP even or a CP odd neutral Higgs boson. Decays of both $H_{1}$ and $H_{2}$ into $2 b$ and $2 \tau$ are studied. 
- $Z \rightarrow H_{1} H_{2} \rightarrow H_{1} H_{1} H_{1}$. Only simultaneous decays of all three $H_{1}$ states either into $6 b$ or into $6 \tau$ have been considered.

The corresponding data files are included in the directory EXPCON. They restrict the allowed parameter space of the NMSSM more strongly than within the previous versions v1 and v2 of NMHDECAY. In particular, the constraints on the decay of a SM-like Higgs boson $h$ to $a a$, where $a$ is a CP odd (singlet-like) scalar, are much stronger. Roughly speaking, the only remaining possibilities (unless the $Z-Z$ - $h$ coupling is heavily suppressed) are:

- $86 \mathrm{GeV} \lesssim m_{h} \lesssim 106 \mathrm{GeV}$ and $m_{a} \lesssim 11 \mathrm{GeV}$ (where the $b \bar{b}$ decay of $a$ is impossible, and no constraints from $a \rightarrow \tau \tau$ are available),

- $106 \mathrm{GeV} \lesssim m_{h} \lesssim 114 \mathrm{GeV}$ with $m_{a}$ going up to $m_{h} / 2$.

(It must be noted, however, that LEP constraints are implemented only for individual processes. Combinations of different processes can rule out points in parameter space, that seem to have passed the individual constraints.)

\subsection{Input parameters}

The implicit definitions of the input parameters $\lambda, \kappa, \mu_{\text {eff }}, A_{\lambda}$ and $A_{\kappa}$ in NMHDECAY v3 differ slightly from the ones in NMHDECAY v2: now these are all defined at a common SUSY scale Q2 (essentially the average squark masses of the first two generations, unless $Q 2$ is defined by the user), in the $\overline{D R}$ scheme as all other soft SUSY breaking parameters. The numerical effect concerns essentially $A_{\lambda}$ : in order to reproduce data points obtained with the previous versions of NMHDECAY, $A_{\lambda}$ has to be shifted by an $A_{\text {top }}$ dependend amount (that can be estimated from the RG equation for $A_{\lambda}$ ).

\subsection{Higgs mass}

The calculation of the radiative corrections to the Higgs masses has been reorganised and improved: Now all parameters used by the corresponding subroutine MHIGGS (gauge and Yukawa couplings, $\tan \beta$, trilinear couplings, quark and squark masses, $\mu_{\mathrm{eff}}$ and Higgs vevs) are defined at a scale QSTSB, that corresponds to an average of the right and left stop quark masses. (QSTSB is computed separately from the scale $Q 2$ above, and can be lower.) Corresponding formulas for the radiative corrections have essentially become simpler, except that contributions from gauge boson and light Higgs boson loops have now to be added explicitly. (In the LLA, which is used for the elektroweak corrections, contributions from 
particles with masses $\sim M_{Z}$ to masses of particles of the same order do not involve large logarithms. Now, the results on heavy Higgs masses are more reliable, without negative effects on the precision of the light Higgs Boson mass.)

Inside the subroutine MHIGGS, dominant gaugino loop contributions are now expressed in the form of a shift of $A_{\lambda}$, which also improves the precision of heavy Higgs spectrum.

New radiative corrections, that have not been considered before, are two loop contributions to the lightest Higgs mass involving two powers of large logarithms and electroweak gauge couplings $g_{1 / 2}$ of the form $\sim h_{t}^{4} g_{1 / 2}^{2} \log ^{2}$, where log denotes a potentially large logarithm. The dominant effect of these contributions depends on the chargino/neutralino spectrum, and is described in [17].

\section{The allowed parameter space of the CNMSSM}

In the CMSSM, the present experimental constraints on sparticle and Higgs masses can be satisfied by chosing sufficiently large soft SUSY breaking terms at the GUT scale, and $\tan \beta$ not too small. As stated above, the required fine tuning is hidden through the implicit determination of $\mu$ and $B$.

In the CNMSSM (without unification of the soft singlet mass, see above) it is often necessary, in addition, to chose a non-universal value for $A_{\kappa}$ in order obtain positive masses squared for both $\mathrm{CP}$ even and $\mathrm{CP}$ odd singlet scalars, even when they are essentially decoupled. Otherwise, for $\lambda \lesssim 0.15$, the allowed parameter space of the CNMSSM corresponds essentially to the one of the CMSSM (except for the possibility to have $m_{h}$ below $114 \mathrm{GeV}$ and unconventional Higgs decay modes, see below).

Larger values for $\lambda$ are not always allowed within the CNMSSM, however; possible problems arise typically in the $\mathrm{CP}$ even Higgs sector: on the one hand, non-vanishing $\lambda$ increases the mass of the lightest Higgs doublet, at tree level, by an amount

$$
\Delta m_{h}^{2}=2 \lambda^{2} M_{Z}^{2} \sin ^{2} 2 \beta /\left(g_{1}^{2}+g_{2}^{2}\right) .
$$

This increase is relevant, however, only for small $\tan \beta$. On the other hand non-vanishing $\lambda$ induces mixings between the doublet and the singlet states. Since the singlet state is typically quite heavy, this mixing reduces the mass of the lightest eigenstate of the $3 \times 3 \mathrm{CP}$ even mass matrix. This lightest eigenstate will then often violate bounds from LEP [7], in spite of its singlet component.

In order to maximize the mass of the lightest $\mathrm{CP}$ even mass eigenstate, this singletdoublet mixing has to vanish (as described in [17]), which implies a relation between $\mu_{\mathrm{eff}}$, 


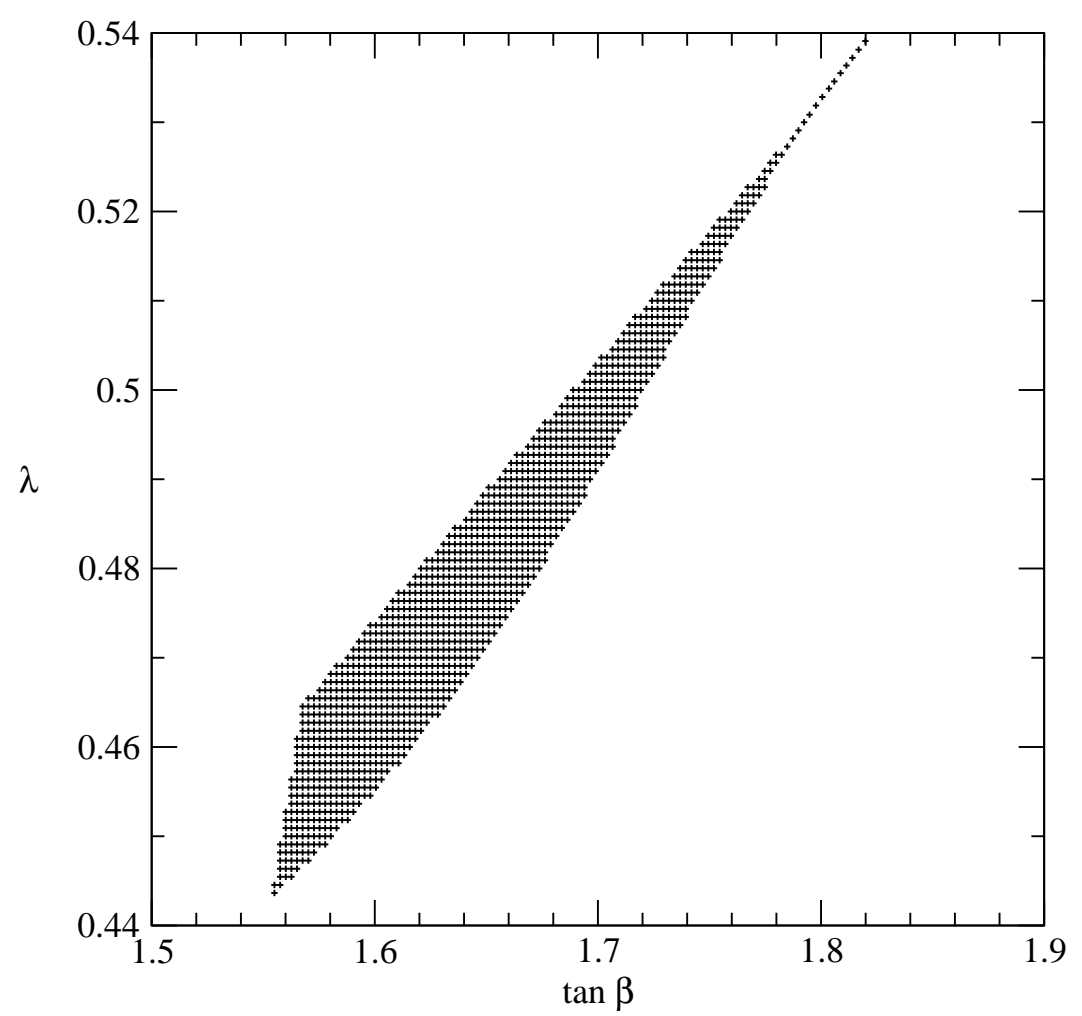

Figure 1: Allowed values for $\lambda$ vs. $\tan \beta$ for $M_{1 / 2}=m_{0}=500 \mathrm{GeV}, A_{0}=-800 \mathrm{GeV}$, $A_{\kappa}=-1500 \mathrm{GeV}$.

$A_{\lambda}, \lambda, \tan \beta$ and $\kappa$. This relation is generally not satisfied within the CNMSSM; then - at least for larger values of $\tan \beta$ - this mixing effect disallows often large values of $\lambda$.

For given soft SUSY breaking terms at the GUT scale, one then obtains typically allowed "islands" in the plane $\lambda$ vs. $\tan \beta$. An example is given in the Figure 1 below, which shows such an allowed island for $M_{1 / 2}=m_{0}=500 \mathrm{GeV}, A_{0}=-800 \mathrm{GeV}, A_{\kappa}=-1500 \mathrm{GeV}$ and $\mu_{\text {eff }}>0$ (with $m_{\text {top }}=175 \mathrm{GeV}$ ).

The lightest (doublet-like) Higgs mass is $\sim 114 \mathrm{GeV}$ near both ends of this region, and up to $\sim 117.5 \mathrm{GeV}$ near its center. All three Yukawa couplings $\lambda, \kappa$ and $h_{t}$ are relatively large, and the region is bounded - apart from LEP constraints on $m_{h}$ - by the condition on the absence of a Landau singularity in the Yukawa sector below $M_{\mathrm{GUT}}$.

Larger values of $\lambda$ are also possible for larger values of $\tan \beta$. Then, however, the lightest (still doublet-like) Higgs mass falls below $114 \mathrm{GeV}$, and $A_{\kappa}$ has to be chosen within a relatively narrow window in order to generate a light (singlet-like) CP odd scalar into which $h$ can decay $[13,14]$. Such a line of allowed values for $A_{\kappa}$ vs. $\lambda$ is shown in Figure 2, for $\tan \beta=5, M_{1 / 2}=m_{0}=500 \mathrm{GeV}$ and $A_{0}=-800 \mathrm{GeV}$. 




Figure 2: Allowed values for $A_{\kappa}$ vs. $\lambda$ for $\tan \beta=5, M_{1 / 2}=m_{0}=500 \mathrm{GeV}$ and $A_{0}=$ $-800 \mathrm{GeV}$.

For $\lambda \lesssim 0.16$, this line joins the CMSSM-like region where $m_{h}>114 \mathrm{GeV}$. Along the line, $m_{h}$ decreases to $m_{h} \sim 86 \mathrm{GeV}$ for $\lambda \sim .4$. Larger values of $\lambda$, i.e. lower values of $m_{h}$, are excluded by LEP. Across the line, the mass of the singlet-like CP odd scalar goes up to $m_{h} / 2$ for $\lambda \lesssim .27$ (where $m_{h} \gtrsim 106 \mathrm{GeV}$ ), but $m_{a} \lesssim 11 \mathrm{GeV}$ for larger values of $\lambda$ (where $\left.m_{h} \lesssim 106 \mathrm{GeV}\right)$; a larger value for $m_{a}$ would imply that $a$ decays mainly into $b \bar{b}$, which is excluded by LEP. The width of the line, expressed in terms of $A_{\kappa}$, is $\sim 1.7 \mathrm{GeV}$ for $\lambda \lesssim .27$, but shrinks down to $\sim 100 \mathrm{MeV}$ for larger values of $\lambda$. This allows to estimate the fine tuning in $A_{\kappa}$ required for this region of the parameter space of the CNMSSM.

In principle, the width of the line (and hence the required relative fine tuning) can depend on all other soft SUSY breaking terms. In order to see this, we have chosen smaller values for $M_{1 / 2}=200 \mathrm{GeV}, m_{0}=100 \mathrm{GeV}$, fixed $\lambda=0.2$ (still at $\tan \beta=5$ ), and plotted the allowed values for $A_{\kappa}$ vs. $A_{0}$ in Figure 3.

In Figure 3, $m_{h} \sim 90 \mathrm{GeV}$ near $A_{0} \sim-200 \mathrm{GeV}$ and increases up to $\sim 112 \mathrm{GeV}$ for $A_{0} \sim-700 \mathrm{GeV}$. (For values of $A_{0} \lesssim 700 \mathrm{GeV}$ one finds $m_{\widetilde{t}_{1}} \lesssim 100 \mathrm{GeV}$, which is excluded by stop searches at LEP). The mass of the singlet-like $\mathrm{CP}$ odd scalar is again $\lesssim 11 \mathrm{GeV}$ for 


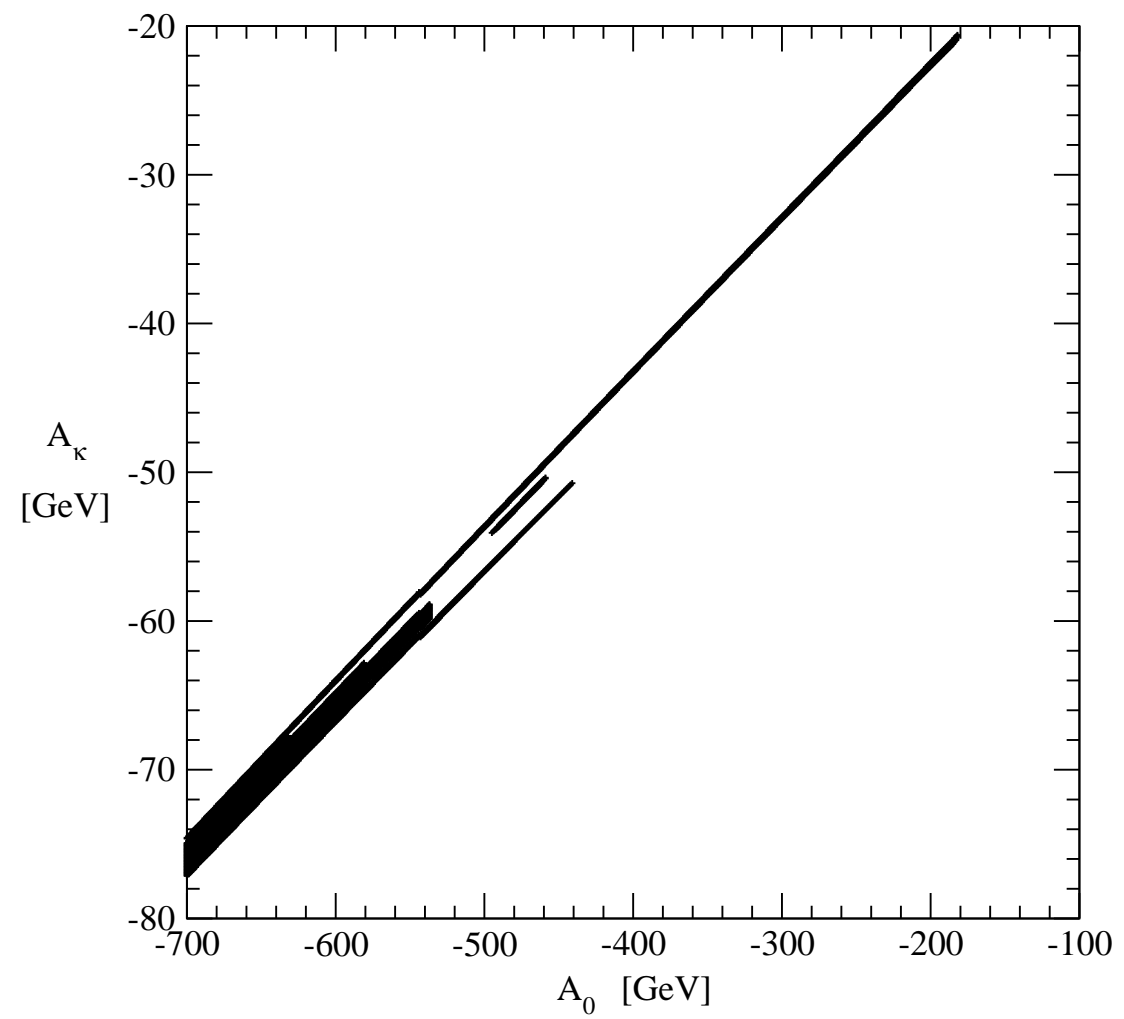

Figure 3: Allowed values for $A_{\kappa}$ vs. $A_{0}$ for $\tan \beta=5, M_{1 / 2}=200 \mathrm{GeV}, m_{0}=100 \mathrm{GeV}$ and $\lambda=0.2$.

$A_{0} \gtrsim-440 \mathrm{GeV}$, but varies from a few $\mathrm{GeV}$ up to $m_{h} / 2$ for $A_{\kappa} \lesssim-440 \mathrm{GeV}$. The width of the line, expressed in terms of $A_{\kappa}$, is now $\sim 2.5 \mathrm{GeV}$ for $A_{0} \sim-700 \mathrm{GeV}$, i.e. the relative fine tuning in $A_{\kappa}$ is somewhat less than before. According to our preliminary analysis, this width cannot be increased by chosing different values of $\tan \beta$.

On the other hand allowed regions in parameter space with $m_{h}<114 \mathrm{GeV}$ remain present also for smaller values of $\lambda$ (at least down to $\lambda \sim 0.1$ ). The present analysis of the allowed parameter space of the CNMSSM is far from complete, but NMSPEC will allow for more detailed studies in this direction in the future. In any case it is important to note that the region in the parameter space of the NMSSM where the lightest SM-like Higgs boson decays dominantly into two CP odd scalars remains present in the CNMSSM; we recall that it could then be quite challenging to detect just one Higgs boson at the LHC [13]. 


\section{Acknowledgement}

We thank P. Bechtle for providing us with the data files containing present constraints on Higgs masses and couplings as obtained by the LEP Higgs Working group. We also thank A. Pukhov for help concerning the development of a simple and global compilation procedure, including all micrOMEGAs_2.0 subroutines. We acknowledge support by the ANR grant PHYS@COL\&COS. 


\section{Appendix: How to use NMSSMTools}

The package NMSSMTools_1.0 contains NMSPEC, the new version of NMHDECAY (v3), a NMSSM version of micrOMEGAs_2.0 and an updated version of the LEP constraints. It can be downloaded from the web page: http://www.th.u-psud.fr/NMHDECAY/nmssmtools.html. Once this compressed tar file is downloaded, one has to type

tar -zxvf NMSSMTools_1.tgz

which will create the directory NMSSMTools_1.0. Inside this directory one finds:

- the directory sources, that contains the subroutines common to NMHDECAY and NMSPEC, the directory micromegas_2.0.3 with the subroutines for the computation of the dark matter relic density, and the directory EXPCON with data files used for the experimental constraints;

- the main programs for NMHDECAY and NMSPEC: nmhdecay.f, nmhdecay_rand.f, nmspec_grid.f, nmspec.f, nmspec_rand.f and nmspec_grid.f;

- six sample input files: inp.dat, randinp.dat, gridinp.dat, inpsp.dat, randinpsp.dat and gridinpsp.dat. All input (and output) files are in SLHA format [18], including some NMSSM specific switches that are described in $[4,5,19]$. We have added privately defined switches for the boundary values of the parameters to be scanned over, the number of points to be scanned over, and an integer ISEED that serves as input for the random number generator. A sample input file randinpsp.dat is given in Table 1;

- corresponding output files: spectr.dat, decay.dat, omega.out, randerr.dat, randout.dat, griderr.dat, gridout.dat, spectrsp.dat, decaysp.dat, omegasp.dat, outsp.dat, randerrsp.dat, randoutsp.dat, griderrsp.dat, gridoutsp.dat;

- A master Makefile.

In order to compile the subroutines in the directories sources and micromegas_2.0.3, one has to type first:

make init

Then to compile the main programs, type:

make 
This will create 6 executable files: nmhdecay, nmhdecay_rand, nmhdecay_grid, nmspec, nmspec_rand and nmspec_grid. Now the user has the following options:

- To study a single point in parameter space, with input parameters at the SUSY scale: Edit the input file inp.dat. Type nmhdecay. The output spectrum is in the file spectr.dat, the branching ratios of the 6 Higgs states in the NMSSM are in the file decay.dat. The dark matter relic density (if OMGFLAG $=1$ in inp.dat) is in the file omega.dat. (A first call of the computation of the dark matter relic density provokes the compilation of additional subroutines of micrOMEGAs_2.0.)

- To scan over randomly chosen points within specified boundary values, with input parameters at the SUSY scale: Edit the input file randinp.dat. Type nmhdecay_rand. The number of points that have passed all tests and their ranges are in the file randerr.dat. The output is in the file randout.dat. In order to specify the output, you need to edit the file nmhdecay_rand.f which contains the subroutine OUTPUT. At the beginning of this subroutine, you find the different elements of the array RES, whose elements are printed into the output file randout.dat. You can declare the elements of the array RES according to your needs; note that the input parameters are elements of the array PAR whose meaning is given at the beginning of the MAIN program in nmhdecay_rand.f. After saving a modified version of nmhdecay_rand.f it is necessary to type make again.

- To scan over a lattice of points within specified boundary values, with input parameters at the SUSY scale: Edit the input file gridinp.dat. Type nmhdecay_grid. The number of points that have passed all tests and their ranges are in the file griderr.dat. The output is in the file gridout.dat. In order to specify the output, you need to edit the subroutine OUTPUT in the file nmhdecay_grid.f, and proceed as described in the case of a random scan above.

- To study a single point in parameter space, with input parameters at the GUT scale: Edit the input file inpsp.dat. Type nmspec. The output spectrum is in the file spectrsp.dat, the branching ratios of the 6 Higgs states in the NMSSM are in the file decaysp.dat. The dark matter relic density (if OMGFLAG $=1$ in inp.dat) is in the file omegasp.dat. The SUSY scale parameters are written in the file outsp.dat in the same format as the file inp.dat, so that outsp.dat can be used as an input file for nmhdecay.

- To scan over randomly chosen points within specified boundary values, with input parameters at the GUT scale: Edit the input file randinpsp.dat (cf table 1). Type 
nmspec_rand. The number of points that have passed all tests and their ranges are in the file randerrsp.dat. The output is in the file randoutsp.dat. In order to specify the output, you need to edit the subroutine OUTPUT in the file nmspec_rand.f, and proceed as described above.

- To scan over a lattice of points within specified boundary values, with input parameters at the GUT scale: Edit the input file gridinpsp.dat. Type nmspec_grid. The number of points that have passed all tests and their ranges are in the file griderrsp.dat. The output is in the file gridoutsp.dat. In order to specify the output, you need to edit the subroutine OUTPUT in the file nmspec_grid.f, and proceed as described above.

To delete all the already compiled codes, the user should type:

make clean 


\begin{tabular}{|c|c|c|}
\hline \multicolumn{3}{|c|}{ BLOCK MODSEL } \\
\hline 3 & 1 & \# NMSSM PARTICLE CONTENT \\
\hline \multicolumn{3}{|c|}{ BLOCK SMINPUTS } \\
\hline 1 & $127.92 \mathrm{D} 0$ & \# ALPHA_EM^-1(MZ) \\
\hline 2 & 1. 16639D-5 & \# GF \\
\hline 3 & $0.1172 \mathrm{DO}$ & \# ALPHA_S(MZ) \\
\hline 4 & 91.187D0 & \# MZ \\
\hline 5 & $4.214 \mathrm{DO}$ & \# MB (MB), RUNNING B QUARK MASS \\
\hline 6 & 175.D0 & \# TOP QUARK POLE MASS \\
\hline 7 & 1.777Do & \# MTAU \\
\hline \multicolumn{3}{|c|}{ BLOCK MINPAR } \\
\hline 0 & 0 & \# REN.SCALE \\
\hline 4 & 1. & \# SIGMU \\
\hline 17 & 100 & \# MOMIN \\
\hline 18 & 100 & \# MOMAX \\
\hline 27 & 200 & \# M12MIN \\
\hline 28 & 200 . & \# M12MAX \\
\hline 37 & 5.0 & \# TBMIN \\
\hline 38 & 5.0 & \# TBMAX \\
\hline 57 & -700 & \# AOMIN \\
\hline 58 & -550 & \# AOMAX \\
\hline \multicolumn{3}{|c|}{ BLOCK EXTPAR } \\
\hline 617 & $.2 \mathrm{D} 0$ & \# LMIN \\
\hline 618 & $.2 \mathrm{DO}$ & \# LMAX \\
\hline 647 & -80 & \# A_KMIN \\
\hline 648 & -55 & \# A_KMAX \\
\hline \multicolumn{3}{|c|}{ BLOCK STEPS } \\
\hline 0 & 1000 & \# NTOT \\
\hline 1 & -1 & \# ISEED \\
\hline
\end{tabular}

Table 1: The randinpsp.dat file for sample parameter scan. 


\section{References}

[1] H. P. Nilles, M. Srednicki and D. Wyler, Phys. Lett. B 120 (1983) 346;

J. M. Frere, D. R. T. Jones and S. Raby, Nucl. Phys. B 222 (1983) 11;

J. P. Derendinger and C. A. Savoy, Nucl. Phys. B 237 (1984) 307;

J. R. Ellis, J. F. Gunion, H. E. Haber, L. Roszkowski and F. Zwirner, Phys. Rev. D 39 (1989) 844;

M. Drees, Int. J. Mod. Phys. A 4 (1989) 3635;

F. Franke and H. Fraas, Int. J. Mod. Phys. A 12 (1997) 479 [arXiv:hep-ph/9512366].

[2] T. Elliott, S. F. King and P. L. White, Phys. Lett. B 351 (1995) 213 [arXiv:hep$\mathrm{ph} / 9406303]$;

U. Ellwanger, M. Rausch de Traubenberg and C. A. Savoy, Phys. Lett. B 315 (1993) 331 [arXiv:hep-ph/9307322], Z. Phys. C 67 (1995) 665 [arXiv:hep-ph/9502206] and Nucl. Phys. B 492 (1997) 21 [arXiv:hep-ph/9611251];

S. F. King and P. L. White, Phys. Rev. D 52 (1995) 4183 [arXiv:hep-ph/9505326];

U. Ellwanger and C. Hugonie, Eur. Phys. J. C 25 (2002) 297 [arXiv:hep-ph/9909260].

[3] P. Skands et al., "A repository for beyond-the-standard-model tools," FERMILABCONF-05-521-T, Presented at Les Houches Workshop on Physics at TeV Colliders, Les Houches, France, 2-20 May 2005, http://www.ippp.dur.ac.uk/montecarlo/BSM/.

[4] U. Ellwanger, J. F. Gunion and C. Hugonie, JHEP 0502 (2005) 066, [arXiv:hepph/0406215], http://www.th.u-psud.fr/NMHDECAY/nmhdecay.html.

[5] U. Ellwanger and C. Hugonie, Comput. Phys. Commun. 175 (2006) 290 [arXiv:hep$\mathrm{ph} / 0508022]$.

[6] U. Ellwanger and C. Hugonie, Phys. Lett. B 623 (2005) 93 [arXiv:hep-ph/0504269].

[7] ALEPH, DELPHI, L3 and OPAL Collaborations and LEP Working Group for Higgs Boson Searches (S. Schael et al.), Eur. Phys. J. C 47 (2006) 547 [arXiv:hep-ex/0602042].

[8] C. Panagiotakopoulos and A. Pilaftsis, Phys. Rev. D 63 (2001) 055003 [arXiv:hepph/0008268]; 
A. Dedes, C. Hugonie, S. Moretti and K. Tamvakis, Phys. Rev. D 63 (2001) 055009 [arXiv:hep-ph/0009125];

A. Menon, D. E. Morrissey and C. E. M. Wagner, Phys. Rev. D 70 (2004) 035005 [arXiv:hep-ph/0404184];

S. J. Huber, T. Konstandin, T. Prokopec and M. G. Schmidt, Nucl. Phys. B 757 (2006) 172 [arXiv:hep-ph/0606298].

[9] M. Cvetic, D. A. Demir, J. R. Espinosa, L. L. Everett and P. Langacker, Phys. Rev. D 56 (1997) 2861 [Erratum-ibid. D 58 (1998) 119905] [arXiv:hep-ph/9703317];

P. Langacker and J. Wang, Phys. Rev. D 58 (1998) 115010 [arXiv:hep-ph/9804428];

J. Erler, P. Langacker and T. j. Li, Phys. Rev. D 66 (2002) 015002 [arXiv:hep$\mathrm{ph} / 0205001]$;

D. E. Morrissey and J. D. Wells, Phys. Rev. D 74 (2006) 015008 [arXiv:hep-ph/0512019].

[10] S. F. King, S. Moretti and R. Nevzorov, Phys. Rev. D 73 (2006) 035009 [arXiv:hep$\mathrm{ph} / 0510419]$.

[11] V. Barger, P. Langacker, H. S. Lee and G. Shaughnessy, Phys. Rev. D 73 (2006) 115010 [arXiv:hep-ph/0603247].

[12] D. J. Miller, R. Nevzorov and P. M. Zerwas, Nucl. Phys. B 681 (2004) 3 [arXiv:hep$\mathrm{ph} / 0304049]$.

[13] B. A. Dobrescu, G. Landsberg and K. T. Matchev, Phys. Rev. D 63 (2001) 075003 [arXiv:hep-ph/0005308];

B. A. Dobrescu and K. T. Matchev, JHEP 0009 (2000) 031 [arXiv:hep-ph/0008192];

U. Ellwanger, J. F. Gunion and C. Hugonie, JHEP 0507 (2005) 041 [arXiv:hep$\mathrm{ph} / 0503203]$;

S. Moretti, S. Munir and P. Poulose, "Another step towards a no-lose theorem for NMSSM Higgs discovery at the LHC," arXiv:hep-ph/0608233;

T. Stelzer, S. Wiesenfeldt and S. Willenbrock, "Higgs at the Tevatron in Extended Supersymmetric Models," arXiv:hep-ph/0611242.

[14] R. Dermisek and J. F. Gunion, Phys. Rev. Lett. 95 (2005) 041801 [arXiv:hepph/0502105], Phys. Rev. D 73 (2006) 111701 [arXiv:hep-ph/0510322] and "The NMSSM 
close to the R-symmetry limit and naturalness in $\mathrm{h} \rightarrow$ aa decays for $\mathrm{m}(\mathrm{a})<2 \mathrm{~m}(\mathrm{~b})$," arXiv:hep-ph/0611142;

S. Chang, P. J. Fox and N. Weiner, JHEP 0608 (2006) 068 [arXiv:hep-ph/0511250] and "Visible cascade Higgs decays to four photons at hadron colliders," arXiv:hep$\mathrm{ph} / 0608310$.

[15] G. Belanger, F. Boudjema, A. Pukhov and A. Semenov, "micrOMEGAs 2.0: A program to calculate the relic density of dark matter in a generic model," arXiv:hep-ph/0607059.

[16] G. Belanger, F. Boudjema, C. Hugonie, A. Pukhov and A. Semenov, JCAP 0509 (2005) 001 [arXiv:hep-ph/0505142].

[17] U. Ellwanger, C. Hugonie, "The upper bound on the lightest Higgs mass in the NMSSM revisited", [arXiv:hep-ph/0612133]

[18] P. Skands et al., JHEP 0407 (2004) 036 [arXiv:hep-ph/0311123].

[19] B. C. Allanach et al., "Les Houches 'Physics at TeV colliders 2005' Beyond the standard model working group: Summary report," arXiv:hep-ph/0602198. 